\title{
Reg upon Act Normal T-cell Exprd Secrtd Measurement
}

National Cancer Institute

\section{Source}

National Cancer Institute. Reg upon Act Normal T-cell Exprd Secrtd Measurement. NCI Thesaurus. Code C81957.

The determination of the amount of the RANTES (regulated on activation, normally, T cell expressed, and secreted) chemokine present in a sample. 